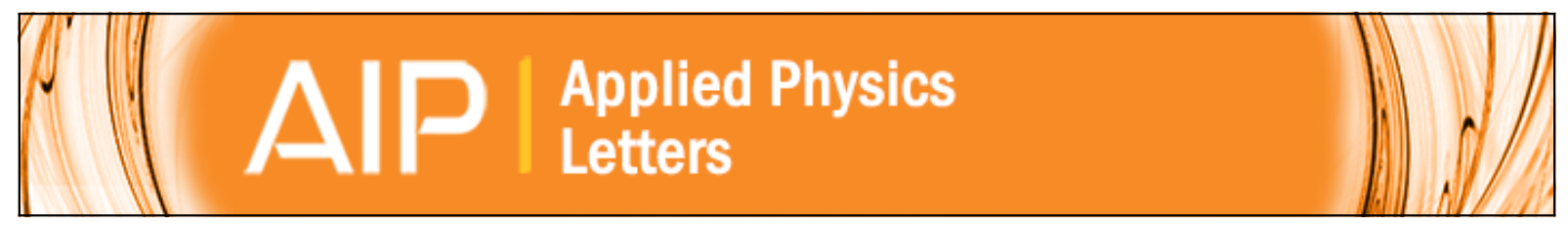

\title{
Effects of depletion on the emission from individual InGaN dots
}

B. Sherliker, M. P. Halsall, P. D. Buckle, P. J. Parbrook, and T. Wang

Citation: Applied Physics Letters 88, 122115 (2006); doi: 10.1063/1.2186973

View online: http://dx.doi.org/10.1063/1.2186973

View Table of Contents: http://scitation.aip.org/content/aip/journal/apl/88/12?ver=pdfcov

Published by the AIP Publishing

\section{AlP}

\section{Create a profile. \\ Sign up today!}




\title{
Effects of depletion on the emission from individual InGaN dots
}

\author{
B. Sherliker ${ }^{a)}$ and M. P. Halsall \\ Department of Electronic and Electrical Engineering, University of Manchester, Manchester M60 1QD, \\ United Kingdom \\ P. D. Buckle \\ Qinetiq PLC, St. Andrews Road, Malvern WR14 3PS, United Kingdom \\ P. J. Parbrook and T. Wang \\ ESPRC National Centre for III-V Technologies, Department of Electronic and Electrical Engineering, \\ University of Sheffield, Sheffield S3 7RH, United Kingdom
}

(Received 17 October 2005; accepted 10 February 2006; published online 23 March 2006)

\begin{abstract}
We report a photoluminescence (PL) study of the effects of carrier depletion on the electronic states of InGaN quantum dots. Samples were fabricated into mesa devices with top Schottky contacts and back ohmic contacts. Submicrometer apertures were created lithographically. Capacitance-voltage measurements of the devices suggest that the dots are fully depleted when they are unbiased. Micro-PL studies of individual dots show narrow linewidths under zero or reverse bias conditions. Forward biasing of the junction results in broadening of the dot levels due to the populating of nearby conduction band states in the underlying quantum well. (C) 2006 American Institute of Physics. [DOI: 10.1063/1.2186973]
\end{abstract}

Self-organized quantum dot structures (SOQDs) are currently being studied for device applications. InGaN SOQDs have recently been used in the active region of laser diodes to improve their performance. ${ }^{1}$ SOQDs are expected to improve the temperature stability of devices ${ }^{2}$ and have also been used to achieve strong luminescence in devices with high defect densities. ${ }^{3}$ The confinement of excitons due to the quasi-zero-dimensional (OD) nature of InGaN SOQDs has been confirmed via the observation of sharp lines in photoluminescence (PL) spectra. ${ }^{4}$ However, the linewidths reported by workers in the InGaN dot system are broad when compared to other systems. This has been ascribed to several processes including rapid dephasing and the effects of local electric fields. We demonstrate that the depletion of $\mathrm{InGaN}$ dots has a dramatic effect on the observed PL linewidths and that the observed line broadening is due to the interaction between the dot levels and neighboring deep donor levels.

The sample was grown by metal-organic vapor-phase epitaxy at low pressure using a close-coupled showerhead reactor. A $1 \mu \mathrm{m} \mathrm{GaN} \mathrm{buffer} \mathrm{layer} \mathrm{was} \mathrm{grown} \mathrm{on} c$-plane sapphire, followed by a nominal $1.5 \mathrm{~nm}$ InGaN layer, grown at $740{ }^{\circ} \mathrm{C}$. To allow for PL measurements the sample was capped with $40 \mathrm{~nm}$ of GaN grown without a pause at the InGaN growth temperature, after which the sample was quenched in an ammonia atmosphere.

Large mesa structures were created on the sample surface, with top Schottky junctions allowing control of the Fermi level in the sample by changing the bias across the junction. Conventional lithographic techniques and dry etching were used to create large $\left(\sim 1 \mathrm{~cm}^{2}\right)$ mesas. Etching around the mesa to below the dot layer allowed direct contact to the GaN buffer/nucleation layer. Some $100 \mathrm{~nm}$ of $\mathrm{Ti} / \mathrm{Au}$ was annealed onto the sample to form the bottom ohmic contacts. The highly conducting GaN nucleation layer forms an effective back contact. A $70 \mathrm{~nm}$ thick Ti/Al Schottky layer was then evaporated onto the surface and sub-

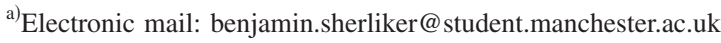

micro meter apertures between 400 and $900 \mathrm{~nm}$ in diameter were formed using electron beam lithography and dry etching. ${ }^{5}$ The devices were characterized by capacitancevoltage measurements that indicate a zero bias depletion layer depth of around $650 \mathrm{~nm}$, corresponding to a net residual $n$-type carrier concentration of $10^{16} \mathrm{~cm}^{3}$, which is fairly typical for undoped GaN. They also indicate a significant series resistance in the undoped $\mathrm{GaN}$ buffer layer making precise knowledge of the barrier potential height difficult. However, the depletion depth was found to reduce to $\sim 350 \mathrm{~nm}$ at $1-2 \mathrm{~V}$ of forward biasing. Micro-PL was recorded with a Renishaw confocal micro-Raman system using the $442 \mathrm{~nm}$ line of a HeCd laser as an excitation source. The sample was mounted on the cold finger of a continuous flow He cryostat and cooled to $4.3 \mathrm{~K}$. The laser spot size was $\sim 2 \mu \mathrm{m}$ and the spectral resolution of the system was $\sim 500 \mu \mathrm{eV}$.

Atomic force microscopy (AFM) studies carried out on an uncapped sample ${ }^{6}$ show that the surface is covered with a very low density $\left(\sim 5 \mathrm{dot} / \mu \mathrm{m}^{2}\right)$ of large nanostructures, with a nonuniform distribution of the background InGaN layer. The average diameter and height of the SOQDs are 30 and $5 \mathrm{~nm}$, respectively. The indium content of this sample is nominally $20 \%$. We note that the AFM images are similar to those seen in other works on InGaN SOQD structures ${ }^{4,7}$ with the exception of the low dot densities. At these densities individual dots can be studied easily with relatively large apertures and low background signals.

In a previous report ${ }^{6}$ we observed (using conventional shadow masks) that the narrowest single exciton lines in a similar sample to the one studied here have a full width half maximum (FWHM) of $\sim 5 \mathrm{meV}$. The slightly larger SOQDs studied in this letter have larger linewidths, with a minimum FWHM of $\sim 8 \mathrm{meV}$. The large linewidths of the single dot emission in this system were described by other workers. ${ }^{4}$ In the InGaAs dot system linewidths are typically more than an order of magnitude smaller. The line broadening has been generally ascribed to spectral diffusion processes $^{8}$ whereby 


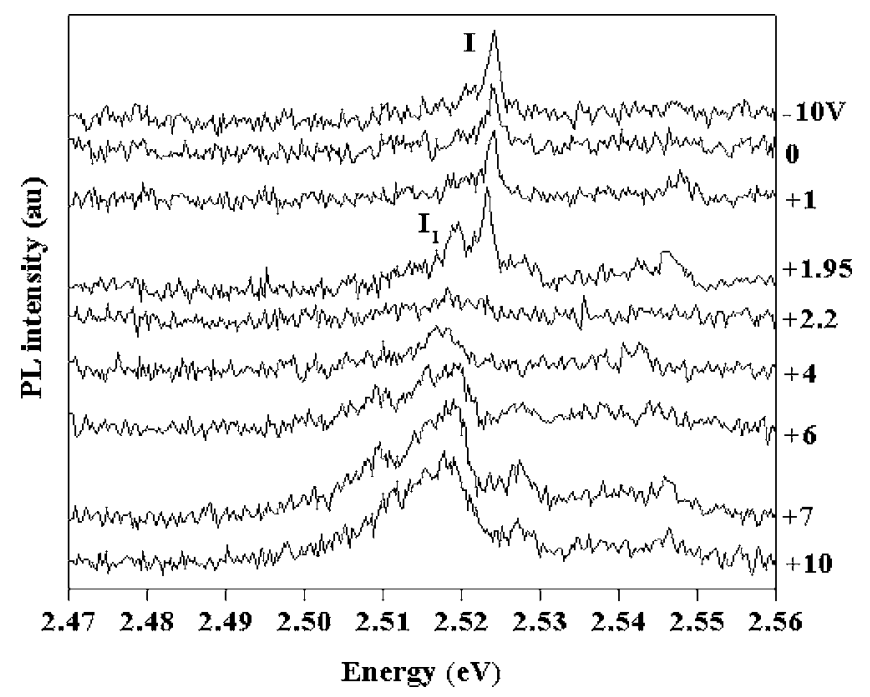

FIG. 1. Micro-PL spectra from a single InGaN SOQD, recorded at $4.3 \mathrm{~K}$ with an applied bias.

the (time-dependent) geometry of nearby charges influences the recombination energy. This gives rise to rapid fluctuations of the peak energy and broadened spectral lines. Similar processes have been observed in GaInP quantum dots where line broadening due to many body interactions in dots filled by residual electrons (perhaps bound to defects) has been reported. ${ }^{9}$ This effect was clearly demonstrated by depleting the GaInP dots under electrical biasing. ${ }^{10}$

The ground state emission from our dots is observed at around 2.4-2.5 eV, while the peak in the emission due to the underlying InGaN quantum well occurs at $2.6-2.7 \mathrm{eV}$. The band gap of $\operatorname{In}_{0.2} \mathrm{Ga}_{0.8} \mathrm{~N}$ is $2.9 \mathrm{eV}$. Thus both the well and dot emission are below the InGaN band edge as expected for nanostructures containing very large piezoelectric fields. Figure 1 shows a series of micro-PL spectra at different applied biases. The spectra were taken with very low power $\left(\sim 2 \mathrm{~W} / \mathrm{cm}^{2}\right)$ excitation, creating carriers directly in the In$\mathrm{GaN}$ quantum well. The integration time was $600 \mathrm{~s}$. The spectra shown were taken when the device was grounded, and even at $0 \mathrm{~V}$ it can be seen that a single line with small linewidth is observed of $\sim 1.5 \mathrm{meV}$, which is much smaller than the average linewidths observed when the sample is not depleted of carriers $(\sim 8 \mathrm{meV})$ and is smaller than the linewidths observed by other workers of $\sim 5 \mathrm{meV}$. ${ }^{11}$ Moriwaki et al. $^{12}$ reported the observation of sharp linewidth $(<200 \mu \mathrm{eV})$ emission from InGaN samples with smaller dots and much higher dot densities. However, in that report the researchers were observing very large numbers $(>20)$ lines from each aperture. At higher excitation powers both line broadening and charged exciton complexes are observed in our samples as will be described in a future publication. However, it is important to point out here that the shifts associated with dot charging are large in this system ${ }^{11}$ due to the screening of the internal fields. Since we only observe a single line in our spectra at low powers and that no charged complexes are seen as a function of bias, we can be sure that the dot is fully depleted at all the biases studied here.

Dephasing broadening has been proposed as a dominating (homogeneous) broadening mechanism for the excitonic emission from the InGaN dot system. ${ }^{12}$ Previously we measured the carrier lifetimes in our system, which were in the nanosecond regime, thus ruling out lifetime broadening as a significant mechanism. ${ }^{6}$ Further evidence for the clear inhomogenous nature of the broadening process in our structures can be seen in the details of the bias dependence of the micro-PL. Under forward bias at around $+2 \mathrm{~V}$ the spectrum changes to show at least one extra line (marked $I_{1}$ ) close in energy to the excitonic emission (marked $I$ ). At higher forward biases the lines broaden considerably and stay redshifted relative to the zero bias line. This effect occurs when the bias is such that one or more neighboring defect states/ localization centers lies very close to the Fermi level with the $I_{1}$ peak and the main peak $(I)$ being ground state excitonic emission for differing geometries of the charge in the vicinity of this state(s). As the Fermi level moves further up under forward biasing more neighboring levels are populated further broadening the emission. Clearly, the indicated biases are across the whole device and the actual voltage across the Schottky junction is difficult to control in the current device due to high contact resistance. However, the effect demonstrates that such devices offer control over the linewidth of the quantum dot emission in this system.

Before discussing the nature of the centers responsible for the observed broadening, it is important to note that we previously reported that the quantum well emission also breaks up into fine spectral lines in micro-PL due to localization centers (alloy fluctuations) whose areal density is around 50 times higher than the dot density. However, the excitonic emission from the quantum dots is to lower energy and is well separated (by more than $100 \mathrm{meV}$ ) from that of these localization centers. Thus the conduction band states in these centers must lie to higher energy and therefore be depleted under all the biases considered here, furthermore if the broadening of the dot levels was due to photocreated carriers in the quantum well then we would not observe a bias dependence. Thus the states involved must be below the dot energy levels, be conduction bandlike, and be optically inactive.

To further study the origin of the state involved we have calculated the potential in the region of the Schottky junction. The conduction band-to-valence band offset ratio in this system is somewhat uncertain, thus we have assumed a 1:1 ratio, which lies approximately in the middle of reported values and an effective electron mass of $0.13 m_{0}$. The barrier potential of the Schottky junction is assumed to be around $0.9 \mathrm{eV}$ as is typical in $\mathrm{GaN},{ }^{13}$ uniform residual doping of the $\mathrm{GaN}$ and an internal electric field of $1.5 \mathrm{MV} / \mathrm{cm}$ have been assumed in the quantum well (i.e., we have assumed pseudomorphic growth). A numerical solution to Schrodinger's equation for the potential formed predicts that the lowest quasibound state of the conduction band of the InGaN quantum well is located very close to the top of the well potential. Figure 2 more clearly shows the micro-PL spectra taken at two different biases, and the inset are calculated Schottky contact potential deduced at two important biases. It can be seen that the broadening is associated with the proximity of the Fermi level to the quantum well conduction band states. The exact nature of the localization of the electron state involved is unclear, but our calculation suggests that the Fermi level is still around $200-300 \mathrm{meV}$ below the quantum well band edge when the broadening mechanism starts to switch on, therefore the most likely candidate is deep donors in the well. Previous studies of Schottky junctions in this system inferred the presence of $V_{N}$ donors at a depth of around $250 \mathrm{meV}$ as an important factor in junction leakage, and 


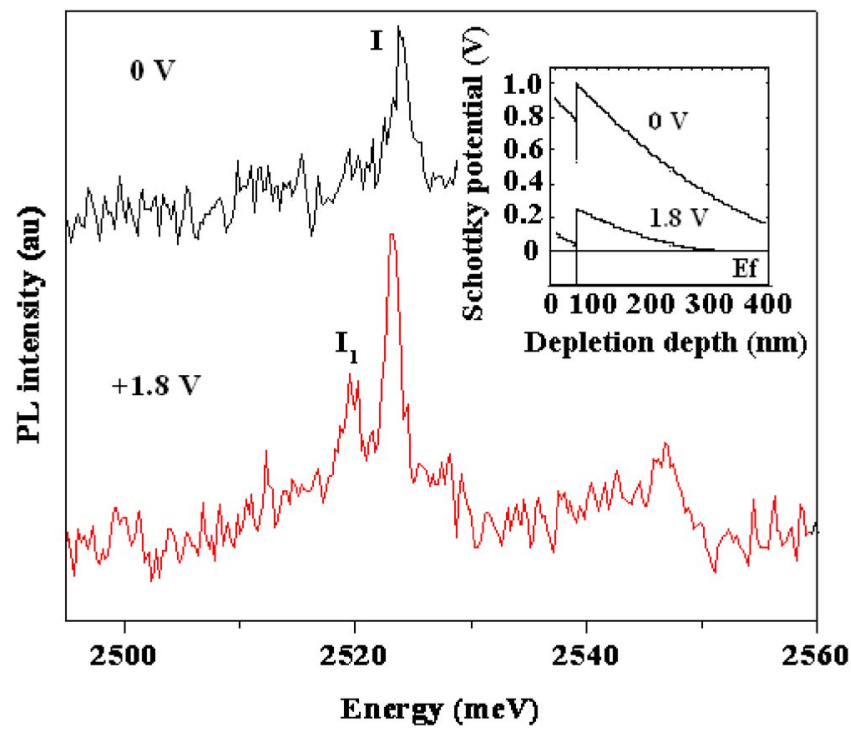

FIG. 2. Micro-PL spectra from a single InGaN SOQD, recorded at $4.3 \mathrm{~K}$ with two different applied biases, also showing calculated Schottky contact potentials.

these may be responsible for the effects described here. It is estimated that the as-grown InGaN quantum well layer has a donor concentration of $\sim 1 \times 10^{17} / \mathrm{cm}^{3}$, which gives an average donor separation of $\sim 20 \mathrm{~nm}$. Thus we expect several donor centers to lie within (or very close to) the dot. Studies on $\mathrm{GaN}$ show that the donor-exciton binding energy is $6-7 \mathrm{meV}$ below the exciton peak. ${ }^{14,15}$ We observe in Fig. 1 that peak $I_{1}$ appears at $+1.95 \mathrm{~V}, \sim 4.5 \mathrm{meV}$ below the excitonic emission, which is consistent with exciton binding to electrons localized at nearby donors. It is impossible to be sure of the nature of these donors, but the fact that we do not observe any apparent charging of the dot itself is consistent with the centers involved being deep levels.
In summary, the bias dependence of the micro-PL indicates that the linewidths of single dots in this system are limited by the residual charge in the vicinity of the dots and that incorporating the dots into Schottky devices can control this, although photocreated carriers can also bind to the impurities in such a way to cause the effect.

${ }^{1}$ Y. Arakawa, IEEE J. 8, 823 (2002).

${ }^{2}$ Y. Arakawa and H. Sakaki, Appl. Phys. Lett. 40, 939 (1982).

${ }^{3}$ B. Damilano, N. Grandjean, J. Massies, and F. Semond, Appl. Surf. Sci. 164, 241-245 (2000)

${ }^{4}$ R. A. Oliver, G. A. D. Briggs, M. J. Kappers, C. J. Humphreys, S. Yasin, J. H. Rice, J. D. Smith, and R. A. Taylor, Appl. Phys. Lett. 83, 755 (2003).

${ }^{5}$ J. J. Finley, P. W. Fry, A. D. Ashmore, A. Lemaitre, A. I. Tartakovskii, R. Oulton, D. J. Mowbray, M. S. Skolnick, M. Hopkinson, P. D. Buckle, and P. A. Maksym, Phys. Rev. B 63, 161305 (2001).

${ }^{6}$ M. P. Halsall, P. Harmer, P. J. Parbrook, and T. Wang, Phys. Status Solidi C 0, 2721 (2003).

${ }^{7}$ K. S. Kim, C. H. Hong, W. H. Lee, C. S. Kim, O. H. Cha, G. M. Yang, E. K. Suh, K. Y. Lim, H. J. Lee, H. K. Cho, J. Y. Lee, and J. M. Seo, MRS Internet J. Nitride Semicond. Res. 5, W11.74 (2000).

${ }^{8}$ J. H. Rice, J. W. Robinson, J. D. Smith, A. Jarjour, R. A. Taylor, R. A. Oliver, G. A. D. Briggs, M. J. Kappers, S. Yasin, and C. J. Humphreys, IEEE Trans. Nanotechnol. 3, 343 (2004).

${ }^{9}$ D. Hessman, P. Castrillo, M. E. Pistol, C. Pryor, and L. Samuelson, Appl. Phys. Lett. 69, 6 (1996).

${ }^{10}$ D. Hessman, J. Persson, M. E. Pistol, C. Pryor, and L. Samuelson, Phys. Rev. B 64, 233308 (2001).

${ }^{11}$ J. W. Robinson, J. H. Rice, K. H. Lee, J. H. Na, R. A. Taylor, D. G. Hasko, R. A. Oliver, M. J. Kappers, C. J. Humphreys, and G. A. D. Briggs, Appl. Phys. Lett. 86, 21 (2005).

${ }^{12}$ O. Moriwaki, T. Someya, K. Tachibana, S. Ishida, and Y. Arakawa, Appl. Phys. Lett. 76, 17 (2000).

${ }^{13}$ H. Hasegawa and S. Oyama, J. Vac. Sci. Technol. B 20, 1647 (2002).

${ }^{14}$ G. E. Bunea, W. D. Herzog, M. S. Unlu, B. B. Goldberg, and R. J. Molnar, Appl. Phys. Lett. 75, 6 (1999).

${ }^{15}$ K. Kyhm, R. A. Taylor, J. F. Ryan, T. Akoi, M. Kuwata-Gonokami, B. Beaumont, and P. Gibart, Phys. Rev. B 65, 193102 (2002). 